operation had failure of the re-repair resulting from recurrent mitral regurgitation in 5 and hemolysis in 1 .

We are grateful to the Editor for the opportunity to further clarify these points.

\author{
Rakesh M. Suri, MD, DPhil \\ Hartzell V. Schaff, MD \\ Cardiovascular Surgery \\ Mayo Clinic College of Medicine \\ Rochester, Minn \\ doi:10.1016/j.jtcvs.2007.03.008
}

\section{Limitations with aprotinin in thoracic aortic surgery: Understanding the clinical outcome beyond bleeding \\ To the Editor:}

I read with great interest the recent article by Dr Sedrakyan and colleagues ${ }^{1}$ detailing their experience with aprotinin in thoracic aortic surgery, using a retrospective casecontrol matching analysis $(\mathrm{n}=168$ [19952003]: deep hypothermic circulatory arrest (DHCA) 64.3\%-67.9\%). The authors conclude in their article that there is American Heart Association level II evidence for aprotinin in thoracic aortic surgery.

I have the following questions for the authors of this excellent study:

1. What was the reexploration rate for bleeding in this case-control series? Did antifibrinolytic exposure make any difference? This outcome variable is an important determinant of mortality after thoracic aortic surgery. ${ }^{2}$ It would be useful to know whether aprotinin was associated with a lower take-back rate.

2. Were anesthetic technique and anesthetic drug doses equivalent in both groups? This is an important determinant of postoperative ventilation time. Was this potential confounder considered before concluding that aprotinin is associated with a decrease in total ventilation time?

3. Were the clinical outcomes, including renal failure and dysfunction, equivalent in the subgroup with DHCA? Our group has recently reported that aprotinin may be associated with renal dysfunction after DHCA. ${ }^{3}$ Does a mixed thoracic aortic cohort explain the differences in these studies?

4. Were there criteria for antifibrinolytic choice in this cohort (eg, previous aprotinin exposure; level of renal impairment)? When was the aprotinin administered (eg, before skin incision or after dissection and cannulation for cardiopulmonary bypass)? It would be useful to understand the dosing style of aprotinin in this study.

5. Were there any hypersensitivity reactions to aprotinin? ${ }^{4}$ Were there any cases of unexpected vascular thrombosis? ${ }^{5}$

I look forward to input from the authors. Again, I congratulate them for a most valuable article on this challenging topic.

John G. T. Augoustides, MD, FASE Department of Anesthesiology and Critical Care Hospital of the University of Pennsylvania Philadelphia, PA

\section{References}

1. Sedrakyan A, Wu A, Sedrakyan G, DienerWest M, Tranquilli M, Elefteriades J. Aprotinin use in thoracic aortic surgery: safety and outcomes. J Thorac Cardiovasc Surg. 2006; 132:909-17.

2. Augoustides JG, Pochettino A, McGarvey ML, Cowie D, Weiner J, Gambone AJ, et al. Clinical predictors for mortality in adults undergoing thoracic aortic surgery requiring deep hypothermic circulatory arrest. Ann Card Anaesth. 2006;9:114-9.

3. Augoustides JG, Pochettino A, Ochroch EA, Cowie D, Weiner J, Gambone AJ, et al. Renal dysfunction after thoracic aortic surgery requiring deep hypothermic circulatory arrest: definition, incidence, and clinical predictors. J Cardiothorac Vasc Anesth. 2006;20:673-7.

4. Beierlein W, Scheule AM, Dietrich W, Ziemer G. Forty years of clinical aprotinin use: a review of 124 hypersensitivity reactions Ann Thorac Surg. 2005;79:741-8.

5. Augoustides JG, Lin J, Gambone AJ, Cheung AT. Fatal thrombosis in an adult after thoracoabdominal aneurysm repair with aprotinin and deep hypothermic circulatory arrest. Anesthesiology. 2005;103:215-6.

doi:10.1016/j.jtcvs.2006.11.071

\section{Reply to the Editor:}

We appreciate Dr Augoustides' kind comments regarding our article. ${ }^{1} \mathrm{We}$ are very much aware of the important contributions he and his colleagues have made on the topic at hand.

We provide the following specific responses to the insightful comments and questions raised in Dr. Augoustides' letter.

1. Three patients required reexploration for bleeding - 1 in the aprotinin group and 2 in the control group.
2. Anesthesia was by a balanced narcotic/inhalation technique in both groups.

3. Renal failure occurred in 3 patients in the control group and 2 in the aprotinin group.

4. Aprotinin was administered after the skin incision. It is our policy to avoid aprotinin in the rare circumstance of recent prior aprotinin exposure.

5. There were no clinically appreciated hypersensitivity reactions to aprotinin. There were no cases of unexpected vascular thrombosis.

In sum, the points raised by Dr Augoustides are all very cogent. On each point, there was, in our study, no evidence of adverse aprotinin-related outcome.

Artyrom Sedrakayan, MD Maryann Tranquilli, $R N$

John A. Elefteriades, $M D$

Department of Cardiothoracic Surgery Yale University School of Medicine, New Haven, CT

\section{Reference}

1. Sedrakayan A, Wu A, Sedrakayan G, et al. Aprotinin use in thoracic aortic surgery: safety and outcomes. J Thorac Cardiovasc Surg. 2006;132:909-17. doi:10.1016/j.jtcvs.2007.01.027

\section{Unilateral as well as bilateral infiltrates should remain part of the definition of pulmonary graft dysfunction}

To the Editor:

We read with interest the article by Oto and associates $^{1}$ in the December 2006 issue of the Journal. The authors underestimate the importance of unilateral infiltrates. We disagree with the statement, "only bilateral infiltrates should be used as part of the definition of primary graft dysfunction" despite their convincing statistical methods. We explain why.

The guidelines of pulmonary graft dysfunction (PGD) $)^{2}$ and validation ${ }^{3}$ thereof is for the clinician to make sense of the data and standardize reporting. The emphasis by the consensus committee on PGD was on providing a definition that could also help in management and prognosis. ${ }^{2}$ PGD is a biological process of reperfusion-ischemic injury redefined with respect to alveolar- 
capillary injury. This process is biological, with reversible and irreversible pathways $^{4,5}$ and along a spectrum that cannot be dichotomous, hence the association of time, arterial oxygen tension/inspired oxygen fraction $\left(\mathrm{PaO}_{2} / \mathrm{FIO}_{2}\right)$ ratios and radiographic findings that are in the context of the International Society of Heart and Lung Transplantation definition. The authors do not reveal their perioperative bronchoscopy protocol, which itself can influence radiographic findings with respect to presence or absence of infiltrates. It is not uncommon that infiltrates could be due to segmental and/or subsegmental mucus plugging, which when it extends to main bronchi can cause significant ventilation-perfusion mismatch and reduction of $\mathrm{PaO}_{2} / \mathrm{FIO}_{2}$ ratios. The population sample, as quite correctly stated by the authors, remains small and heterogeneous; primary pulmonary hypertension is a bilateral problem and requires bilateral lung transplantation. The authors should analyze their findings with respect to a clean cohort of infectious lung disease, for example, to avoid the confounding effect of pulmonary hypertension. The reader will soon realize that the majority of patients with pulmonary hypertension were among the cohort with bilateral infiltrates.

Moreover, patients with fixed pulmonary hypertension are more likely to experience reperfusion-ischemic injury with higher PGD grades. ${ }^{5}$ Recently, aprotinin has been shown to reduce reperfusion injury and allograft dysfunction. It is unclear from the manuscript which antifibrinolytic was administered during transplantationthe type of antifibrinolytic being a potential confounder. The authors state that unilateral infiltrates were associated with PGD grade 3, but that diminished at T48 hours. However, when compared with the absence of infiltrates at T0, it had decreased. It may be more appropriate to look at the absolute difference from T0 to T48 rather than the relative difference, which in a larger homogeneous population sample would minimize confounding. Short of radiographic findings, a clinician can be at loss if $\mathrm{PaO}_{2} /$ $\mathrm{FIO}_{2}$ ratios are the only information provided to make a diagnosis of PGD and/or to intervene with medical therapy or bronchoscopy. Given the therapeutic and diagnostic power of bronchoscopy, any infiltrate on the chest radiograph is of paramount importance in decision making and detection of this biological PGD process. Furthermore, despite the inherently subjective interpretation of a chest radiograph, a transplant physician can recognize patterns of unilateral infiltrates that are typical of the most severe and rapidly progressing to grade $3 \mathrm{PGD}$, which could go unnoticed if only bilateral infiltrates are considered.

\section{Jeffrey Shuhaiber, MD Department of Thoracic and Cardiovascular Surgery Loyola University Medical Center Chicago, Ill}

\section{References}

1. Oto T, Griffiths AP, Levvey BJ, Williams TJ, Snell GI. Unilateral radiographic abnormalities after bilateral lung transplantation: exclusion from the definition of primary graft dysfunction? J Thorac Cardiovasc Surg. 2006; 132:1441-6.

2. Prekker ME, Nath DS, Walker AR, Johnson AC, Hertz MI, Herrington CS, et al. Validation of the proposed International Society for Heart and Lung Transplantation grading system for primary graft dysfunction after lung transplantation. J Heart Lung Transplant. 2006;25:371-8.

3. Shargall Y, Guenther G, Ahya VN, Ardehali A, Singhal A, Keshavjee S, ISHLT Working Group on Primary Lung Graft Dysfunction. Report of the ISHLT Working Group on Primary Lung Graft Dysfunction part VI: treatment. J Heart Lung Transplant. 2005;24: 1489-500. Epub 2005 Jul 27.

4. Bittner HB, Richter M, Kuntze T, Rahmel A, Dahlberg P, Hertz M, et al. Aprotinin decreases reperfusion injury and allograft dysfunction in clinical lung transplantation. Eur J Cardiothorac Surg. 2006;29:210-5.

5. Christie JD, Carby M, Bag R, Corris P, Hertz $\mathrm{M}$, Well D. Report of the ISHLT working group on primary lung graft dysfunction part II: definition. A consensus statement of the International Society for Heart and Lung Transplantation. J Heart Lung Transplant. 2005;24:1454-9

doi:10.1016/j.jtcvs.2007.01.084

\section{Reply to the Editor:}

The aims of our study were to describe the difference of unilateral and bilateral radiographic infiltrates on early posttransplant outcomes, including primary graft dysfunction (PGD) grade. ${ }^{1,2}$ As Shuhaiber states, a perioperative bronchoscopy protocol could influence radiographic findings. In our protocol, inspection and toileting bronchoscopy was routinely performed before donor lung procurement, immediately after implantation, and within 6 hours after admis- sion to the intensive care unit. Therefore, significant airway secretions or mucus plugging were unlikely to be present in the early postoperative period. Moreover, for the purpose of PGD grading, only radiographic infiltrates, consistent with pulmonary edema rather than atelectasis, were assessed (as per the International Society for Heart and Lung Transplantation PGD grading guideline. ${ }^{2}$ ).

Notwithstanding, mucus plugging, clot, or extubation itself ${ }^{3}$ could cause a temporary reduction of arterial oxygen tension/ fraction of inspired oxygen $\left(\mathrm{PaO}_{2} / \mathrm{FIO}_{2}\right) \mathrm{ra}-$ tio. Therefore, the worst $\mathrm{PaO}_{2} / \mathrm{FIO}_{2}$ ratio might not represent true graft function ${ }^{4}$ and should not be used for PGD grading.

Pulmonary hypertension $(\mathrm{PH})$ is a significant risk factor for posttransplant radiographic infiltrates. In our study, the number of patients with $\mathrm{PH}$ was small; thus, the majority of bilateral infiltrates were seen in the non-PH patient group. Moreover, the official PGD grading system applies to both $\mathrm{PH}$ and non-PH recipients.

Antifibrinolytic agents were used for patients having a higher risk of bleeding (eg, cardiopulmonary bypass, previous thoracic operation), patients already at increased risk of postoperative pulmonary infiltrates. Therefore, in this circumstance, the radiographic infiltrates are potentially multifactorial.

Although further study including multivariate analysis is needed, our study clearly showed that the early posttransplant outcome of the unilateral infiltrates was similar to that in the group having a clear chest $\mathrm{x}$-ray film and significantly better than that in those with bilateral infiltrates. Therefore, we believe that in bilateral lung transplantation, only bilateral infiltrates should be used as part of the definition of PGD.

Takahiro Oto, $M D^{a}$

Gregory I. Snell, $M D^{b}$

Department of Thoracic Surgery

Okayama University Hospital ${ }^{a}$

Okayama, Japan

Allergy, Immunology and Respiratory Medicine The Alfred Hospital

Melbourne, Australia

\section{References}

1. Oto T, Griffiths AP, Levvey BJ, Williams TJ, Snell GI. Unilateral radiographic abnormalities following bilateral lung transplantation: exclusion from the definition of primary graft 